\title{
Challenges of Precision Assembly with a Miniaturized Robot
}

\author{
Arne Burisch, Annika Raatz, and Jürgen Hesselbach \\ Technische Universität Braunschweig, \\ Institute of Machine Tools and Production Technology \\ Langer Kamp 19 b, 38106 Braunschweig, Germany \\ a.burisch@tu-bs.de
}

\begin{abstract}
The first part of the paper describes the miniaturized robot Parvus, which is suitable for desktop factory applications. The Parvus is well equipped for pick-and-place of micro parts in precision assembly. The challenges of precision assembly are discussed considering the technical data and behavior of the robot. The hybrid robot operates based on parallel kinematics and is driven by micro harmonic drive gears. Due to its size-reduction, the Parvus offers prospects, but also constraints which are discussed and presented by measuring data. Finally, solutions for improving the precision of the robot are presented.
\end{abstract}

Keywords: Desktop factory, miniaturized robot, micro gripper, micro gear.

\section{Introduction}

Nowadays a trend of miniaturization with regard to product development can be observed in several industrial sectors. The Nexus III market study predicts that the market of millimeter-sized MST-products (Micro System Technology) will grow $16 \%$ per year. However, an increasing gap can be observed concerning the dimensions and costs between the products and the production systems used. Assembly lines and clean rooms for millimeter-sized products often measure some tens of meters and are mostly too expensive for small- and medium-sized businesses. Thus, many microproducts are assembled by hand, which results in high assembly costs that amount to $20 \%$ to $80 \%$ of the total production costs [1]. A solution to prevent this could be the cost-optimized, flexible desktop factory for micro production.

\section{The Miniaturized Robot Parvus}

The robot Parvus, as shown in Figure 1, is a size-adapted handling device using innovative, miniaturized machine parts. With size-adapted handling devices, in the range of several centimeters to a few decimeters, easily scalable and highly flexible production technology can be achieved. The challenge of the functional model Parvus was to develop a miniaturized precision industrial robot with the full functional range of larger models. 
The robot consists of a typical parallel structure, driven by Micro Harmonic Drive [2] gears combined with Maxon electrical motors. This plane parallel structure offers two translational DOF in the $x-y$-plane. The $z$-axis is integrated as a serial axis in the base frame of the robot. The easy handling of the whole plane parallel structure driven in z-direction is possible due to the minimized drive components and light aluminum alloy structure. The rotational hand axis $\Psi$ was designed as a hollow rotational axis to be the Tool Center Point (TCP) of the parallel structure. This allows media such as a vacuum to be passed along the hand axis. This axis, with a diameter of $2.5 \mathrm{~mm}$, can be equipped with several types of vacuum grippers. The development of the Parvus, its fundamentals, the miniaturized drive systems, and the robot design approaches have already been described in several previous papers [3].

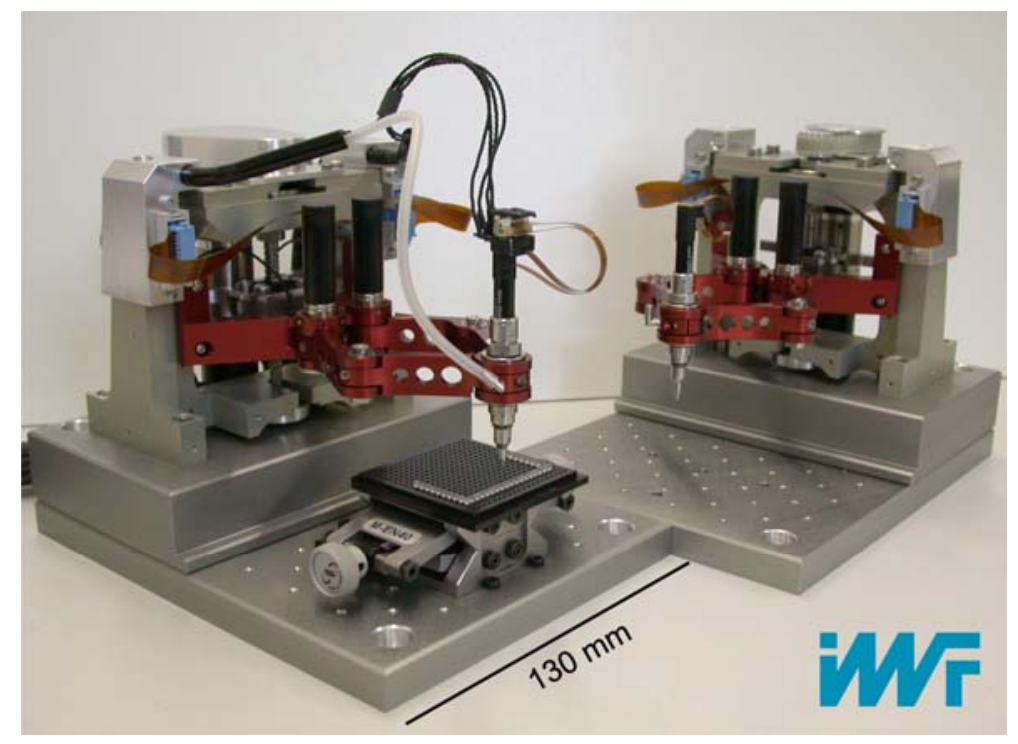

Fig. 1. Two functional models of the miniaturized robot Parvus

\section{Challenges of Precision Assembly}

In most cases, the precision assembly of MST-products needs a highly precise pickand-place application of the related parts. One example is the placement of optical ball lenses in a micro-optical LIGA bench with accuracy in the range of $1 \mu \mathrm{m}$ [4]. This can be achieved by passive alignment and a pick-and-place process using a vacuum gripper. Other parts, such as prisms and photodiodes, can also be mounted in this way. The demonstrators of micro-electric actuators developed within the collaborative research center 516 in Braunschweig and Hannover also need a pick-and-place application of small ruby balls with a diameter of a few hundred micrometers [5].

For these pick-and-place applications, it is essential that the device always reaches the same assembly position multiple times. This is well described and characterized by the repeatability of the handling device following the standard of EN ISO 9283 [6]. 
The repeatability can be taken as a reference value for the maximum position accuracy of the robot end-effector. It is hereby assumed that the robot always reaches the position coming from the same direction.

However, in the case of picking several different parts from magazine trays around the assembly area, the handling device will reach the position from different directions. To get further information about the behavior of the robot in this case, the multiple direction position accuracy [6] has to be taken into account.

Another exemplary scenario is picking a glass ball out of a channel and moving the end-effector precisely along this channel. In this case, good path accuracy [6] of the robot is necessary to move a gripper precisely inside a channel without collision.

\section{Prospects and Constraints of the First Functional Model Parvus}

In most cases of typical pick-and-place applications, the Parvus is well-suited for a high precision assembly process. Figure 2 shows the robot being equipped with a vacuum gripper and a pneumatic mechanical micro gripper [7] for gripping a variety of different micro parts. The repeatability of the robot end-effector was measured within the primary (mainly used) workspace, as shown in Figure 3. The worst value of the repeatability is $5.7 \mu \mathrm{m}$ (at measuring point P3, with 3 Sigma). This measured value is in the range of the expected repeatability of $2-6 \mu \mathrm{m}$, which was simulated within the primary workspace and based on an angular repeatability of $0.0027^{\circ}$ [2] of the micro gears. As described in previous papers, this value was achieved by optimizing the stiffness of the micro gears.
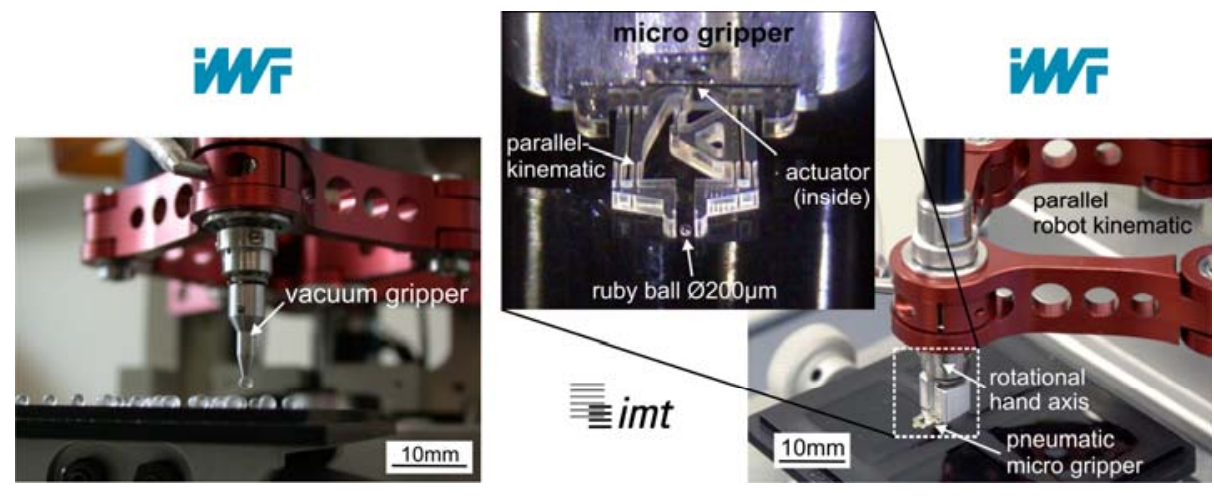

Fig. 2. Parvus with vacuum gripper (left), micro gripper (right)

As stiffness of the micro gears is important for the repeatability of the robot, it is also very important for the multiple direction accuracy. Stiffness related factors, including backlash and hysteresis in the robot drives, have a significant influence on the results. The multiple direction accuracy has been measured within the primary workspace at points P1, P2 and P5. The results show that these values differ 10 - 30 times as much as the repeatability. 


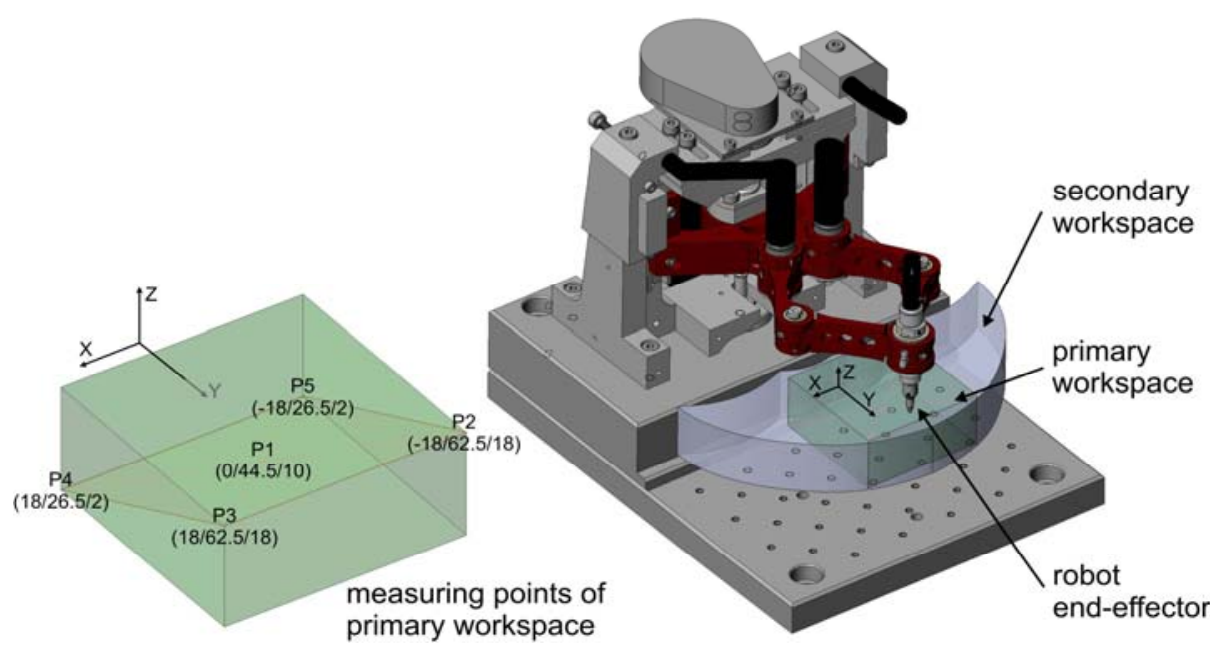

Fig. 3. Workspace and measuring points of Parvus

It has been investigated why there is such a difference between the multiple direction accuracy of the robot and the repeatability, even though the micro gears are backlash free. In this case, the relatively low stiffness of $2.6-6.13 \mathrm{Nm} / \mathrm{rad}[8,2]$ of the micro drives compared to the dimensions of the robot structure causes this effect. When unloaded, the micro gears are free of backlash. However, the robot structure of the Parvus consists of preloaded ball bearings and is directly connected to the micro gears. Ball bearings cause frictional torque in the robot structure and the micro gears. The frictional torque stops the gear before it can reach its neutral position. This effect occurs when approaching from the left or the right side and results in elastic backlash in the combination of robot structure with the micro gears. If there were any backlash in the system, an additional pre-load in the robot structure would reduce this effect and improve the multiple direction accuracy. To prove this assumption, an experiment with preloaded robot structure was carried out, as shown in Figure 4. There was a spring force $F_{\text {spring }}$ applied between the passive joints of the robot structure to induce a reactive torque $M_{\text {gear }}$ in the micro gears. This torque was higher than the frictional torques of the robot structure, which is therefore able to deflect the gear beyond its neutral position into a more stable state in both directions. The results, shown in Figure 5, verify the above mentioned assumption and show that the applied force can improve the multiple direction accuracy by up to 3 times, here from $55 \mu \mathrm{m}$ to $18 \mu \mathrm{m}$. The experiment with the spring is actually possible in a very small area of the workspace. The small range of applicable spring forces between the robot joints, limits the movement of the robot structure. Therefore, different springs have to be used for different points in the workspace. To implement a stable preload on the robot drives without limiting the workspace, the torque has to be applied directly to the drives. Here a torsion spring or an active torque generator has to be connected to the output of the micro gears. 


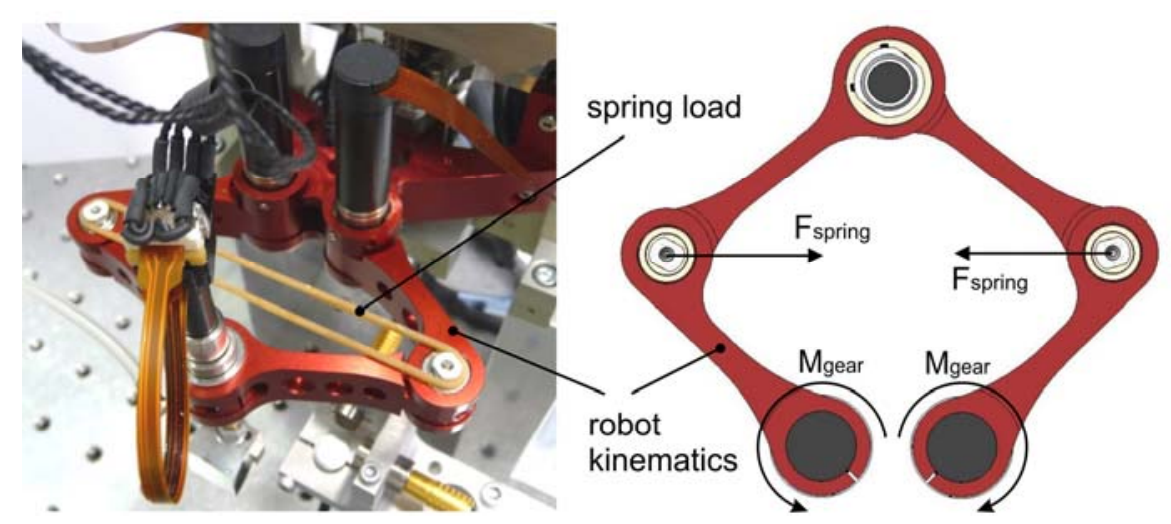

Fig. 4. Robot kinematics with spring load and reaction torque
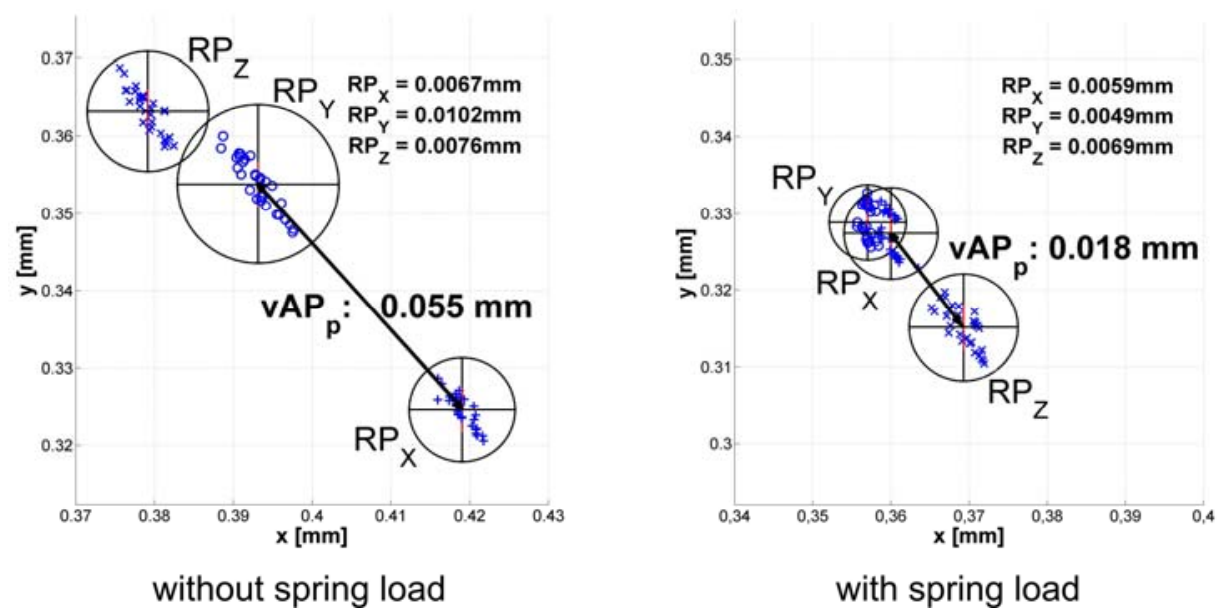

Fig. 5. Results of measured multiple direction accuracy at point P5

In addition, there are also other influences of the micro gears concerning the characteristics of the robot kinematics. In general, harmonic drive gears show special behavior in kinematic error and transmission compliance, as shown in Figure 6 (left) [9]. At the high grade of miniaturization of the micro harmonic drive gears used in the robot, theses effects immensely influence the path accuracy of the entire robot, as shown in Figure 6 (right). In most cases, the path accuracy is only important for machine tools and not for pick-and-place robots. However, in some cases it has to be considered that there is a deviation of the set path.

One example is the automatic ball feeder (designed at IMT of TU Braunschweig), where the Parvus has to move its micro gripper inside a channel for gripping a prepositioned glass ball, as shown in Figure 7. Here the gripper has to move along a relatively straight line, which requires good path accuracy. 


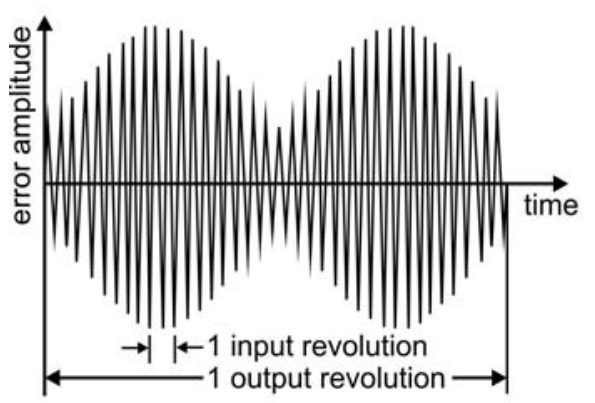

typical transmission error displayed by harmonic drive gears

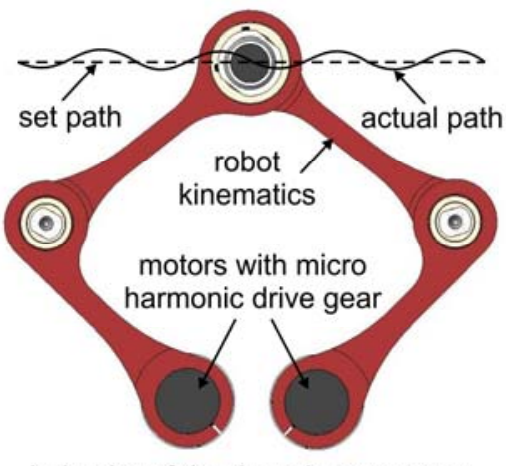

behavior of the the robot structure

Fig. 6. Transmission error of harmonic drive gears [9] and behavior of the Parvus

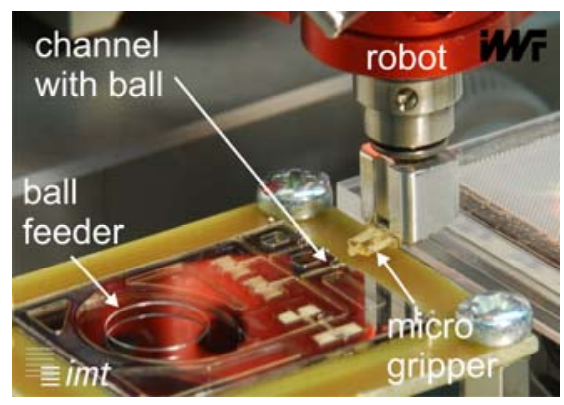

Fig. 7. Automatic ball feeder in interaction with Parvus

To characterize the path accuracy of the robot, a sample path has been measured with a 3D laser tracker. Figure 8 shows the results of the measured path accuracy (left) and the experimental setup (right). The actual path of the Parvus follows a periodic behavior with a maximum deviation of $100 \mu \mathrm{m}$ from the set path. This behavior is obviously caused by the transmission error of the micro gears. The transmission error inside the gear can also be described as a kinematic effect. There cannot occur any dynamic effects, thus the measurements have been done at very low speed.

In order to optimize the path accuracy of the robot, it is necessary to measure the transmission error at the output of each gear. This is necessary because the path accuracy of the robot shows a combination of the transmission errors of both drives of the parallel robot structure. The best way to detect this behavior is using a small and precise angular sensor. If it is possible to obtain precise information about the driving angle during operation, the control could compensate the transmission error of the gears and help improving the path accuracy of the robot. A small precise angular sensor is currently under testing. Another approach is the use of computed feed forward control to reduce disturbing effects coming from the gears. This is under examination using the inverse dynamic model of the Parvus. 

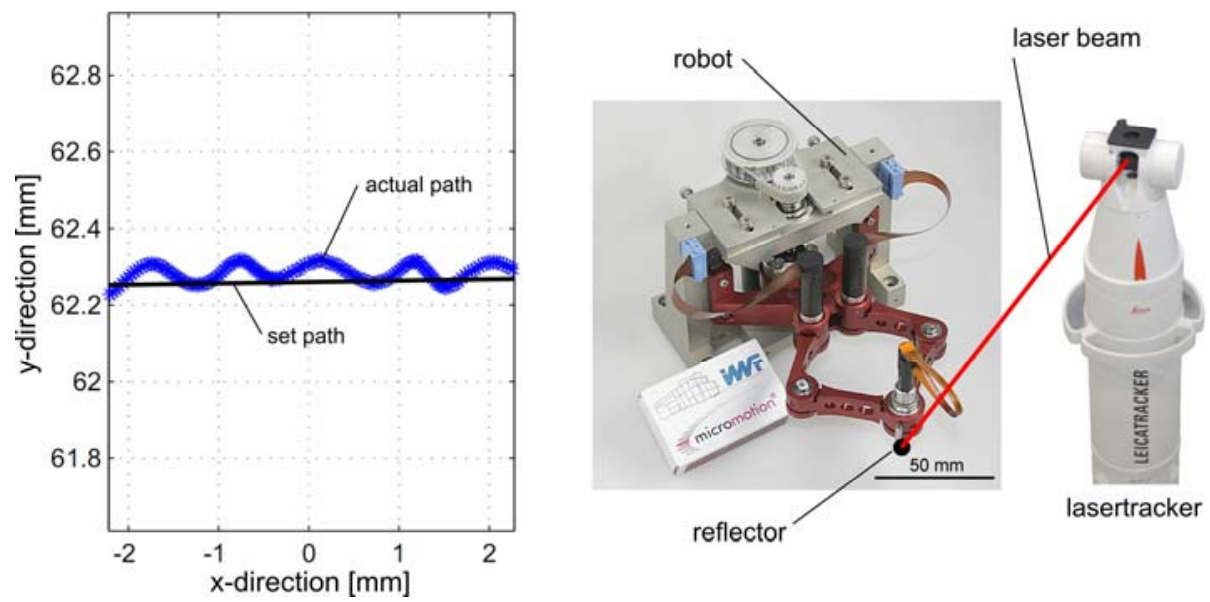

Fig. 8. Measured path accuracy of the Parvus (left), measuring setup (right)

Furthermore, the above mentioned measurements (repeatability, multiple direction accuracy and path accuracy) of the robot kinematics driven by micro harmonic drive gears will also be done with the same robot kinematics connected to small harmonic drive gears RSF-5A. This can help to ensure that there are no disturbing effects as a result of the kinematics and ball bearings.

\section{Conclusions and Outlook}

The experiments and measurements with the Parvus have shown that the robot is well suitable for pick-and-place applications with repeatability exceeding $5 \mu \mathrm{m}$. It has been shown that the stiffness of the micro gears influences the repeatability of the robot. The multiple direction accuracy of the actual functional model still has to be improved. The experiment has demonstrated that preloaded torque can improve this behavior. To ensure this, further experiments have to be performed. A way must be found to implement a stable preload on the robot drives without limiting the workspace. It has been observed that there is a deviation from the set path of the robot. Therefore, path accuracy will be measured and improved. The required hardware and control strategies are currently under testing. The results of measurements and strategies for improving the accuracy of the robot will be used for the design of the next prototype of Parvus.

\section{Acknowledgement}

The authors gratefully acknowledge the funding of the reported work by the German Research Center (Collaborative Research Center 516 "Design and Manufacturing of Active Micro Systems“). 


\section{References}

1. Koelemeijer, S., Jacot, J.: Cost Efficient Assembly of Microsystems. In: MST-news, vol. 1, pp. 30-32. Verlag VDI/VDE Innovation + Technik GmbH (1999)

2. Micromotion GmbH, Catalogue: Precision Microactuators, vol. 04/2005 MM 900118 (2009), http: / /www. micromotion-gmbh. de

3. Burisch, A., Wrege, J., Raatz, A., Hesselbach, J., Degen, R.: PARVUS - miniaturised robot for improved flexibility in micro production. Journal of Assembly Automation, Emerald 27(1), 65-73 (2007)

4. Gerlach, A., Ziegler, P., Mohr, J.: Assembly of hybrid integrated micro-optical modules using passive alignment with LIGA mounting elements and adhesive bonding techniques. In: Microsystem technologies, vol. 7, pp. 27-31. Springer, Berlin (2001)

5. Hesselbach, J., Pokar, G., Wrege, J., Heuer, K.: Some Aspects on the Assembly of Active Micro Systems. Production Engineering. Research and Development 11(1), 159-164 (2004)

6. EN ISO 9283: Industrieroboter: Leistungskenngrößen und zugehörige Prüfmethoden. Verlag, Berlin (1999)

7. Hoxhold, B., Büttgenbach, S.: Micro Tools with Pneumatic Actuators for Desktop Factories. Sensors and Transducers 7(10/09), 160-169 (2009)

8. Slatter, R., Degen, R.: Miniature Zero-Backlash Gears And Actuators For Precision Positioning Applications. In: Proc. of 11th European Space Mechanisms and Tribology Symposium ESMATS 2005, Lucerne, Switzerland, September 21-23, pp. S.9-S.16 (2005)

9. Tuttle, T.D.: Understanding and Modeling the Behavior of a Harmonic Drive Gear Transmission. Technical Report: AITR-1365, Massachusetts Institute of Technology, Cambridge, MA, USA (1992) 